\title{
DHZ-Fortbildungspunkte: Unterstützung durch viele Fachverbände
}

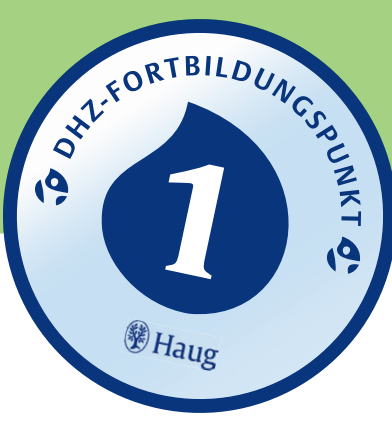

Viele Leser der DHZ sammeln DHZFortbildungspunkte für das freiwillige Fortbildungszertifikat für Heilpraktiker. Seit 2010 unterstützen folgende Fachverbände die FobiPunkte der DHZ als Nachweis für die kontinuierliche Fortbildung an: BDH, AGAHP, BDC, DAGC (Deutsch-Amerikanische Gesellschaft für Chiropraktik e.V.), HP-Gesellschaft für Elektroakupunktur nach Voll e.V., $\mathrm{HPGO}_{3}$.

Heilpraktiker bilden sich sehr engagiert und regelmäßig fort - ganz ohne Zwang und Pflicht, einfach, weil sie wissen, dass sie dies ihren Patienten schuldig sind und eine Praxis sich heutzutage auch kaum anders erfolgreich führen ließe. Doch tatsächlich sind sie nach der Berufsordnung für Heilpraktiker (BOH, Artikel 5) zur ständigen Fortbildung verpflichtet. Diese Fortbildung müssen sie zudem nachweisen - im Extremfall vor Gericht.

Ganz unabhängig davon ist eine kontinuierliche Qualifizierung heutzutage unerlässlich. Nicht nur Politiker und maßgebliche Vertreter des Gesundheitswesens fordern eine dokumentierte Qualitätssicherung. Moderne, aufgeklärte Patienten erwarten ebenfalls - völlig zu Recht - aktualisiertes Wissen und umfassende Kompetenz auf neuestem Stand von ihren Therapeuten.

\section{Fortbildungszertifikate - längst} Alltag in vielen Medizinberufen

Viele medizinische Berufe haben inzwischen ein eingeführtes Fortbildungsprogramm, das kontinuierliches Weiterlernen fordert, dokumentiert, zertifiziert und nicht zuletzt auch das Ansehen des Zertifizierten steigert. Das CME-System (continuing medical education) der Ärzte hat sich weltweit durchgesetzt. Aber auch die Apotheker, pharmazeutisch-technische Assistenten, Pflegekräfte, Physiotherapeuten und einige Heilpraktikerverbände haben die Notwendigkeit der kontinuierlichen Fortbildung erkannt und inzwischen ihre eigenen Fortbildungszertifikate entwickelt. Gerade vor dem Hintergrund der Berufsstandssicherung ist der Nachweis von qualifizierter Fortbildung von zentraler Bedeutung. So bietet z. B. der Bund Deutscher Heilpraktiker (BDH) gemeinsam mit AGAHP, BDC, DAGC, HP-Gesellschaft für Elektroakupunktur nach Voll e.V., $\mathrm{HPGO}_{3}$ ein freiwilliges Fortbildungszertifikat für Heilpraktiker an. Diese Institutionen nutzen die DHZ-Fortbildungspunkte, um ihren Mitgliedern interaktive Fortbildung zu ermöglichen.

\section{DHZ-Punkte sammeln}

\section{für das Zertifikat}

Das freiwillige Fortbildungszertifikat für Heilpraktiker der genannten Fachverbände basiert im Wesentlichen auf dem oben beschriebenen generell anerkannten Fortbildungsmodell (CME) und garantiert so die Akzeptanz durch Öffentlichkeit, andere Gesundheitsberufe und die Politik. Die Grundeinheit dieser Fortbildungsaktivitäten ist der Fortbildungspunkt (FP). Dieser entspricht i. d. R. einer abgeschlossenen Fortbildungsstunde (45 min). Für die erworbenen Fortbildungspunkte muss ein Nachweis erbracht werden. Punkte können z. B. entweder durch den Besuch von Fortbildungsveranstaltungen, Kongressen, Fachtagungen oder Workshops gesammelt oder über sog. interaktive Fachfortbildungen durch Internet oder Fachzeitschriften erworben werden. Bei der interaktiven Fachfortbildung sind der Nachweis der Qualifizierung und die schriftliche Auswertung des Lernerfolgs eine Grundvoraussetzung für die Vergabe der Fortbildungspunkte.

Das Fortbildungszertifikat für Heilpraktiker wird ausgestellt, wenn Sie in 3 Jahren 120 Fortbildungspunkte erworben, diese dokumentiert und einen entsprechenden Antrag auf Zertifizierung bei der Dokumentationsstelle des BDH gestellt haben. Davon können Sie maximal 10 Punkte pro Jahr für das Zertifikat mithilfe der interaktiven Fortbildung durch Fachzeitschriften sammeln (Infos unter www.bdh-online.de).

Das Fortbildungszertifikat steht allen Heilpraktikern offen, unabhängig von ihrer Verbandszugehörigkeit.

\section{Homöopathie-Zertifikat:}

\section{Punkte erwerben mit der DHZ}

Die SHZ ist eine gemeinnützige, von Einzelinteressen unabhängige Stiftung des bürgerlichen Rechts.

Sie hat die Aufgabe, ein hohes Qualitätsniveau in der Aus- und Weiterbildung von Homöopathen (Heilpraktikern und Ärzten) zu fördern und zu sichern, mit dem Ziel, Qualitätskriterien für eine klassisch homöopathische Behandlung in der Öffentlichkeit zu etablieren. Die umfassenden Qualifikationsrichtlinien definieren Inhalt und Umfang homöopathischer Ausbildungen (mind. 550 Unterrichtseinheiten Kontaktstudium und 1250 Unter- 


\section{DHZ-Fortbildungspunkte - Fragebogen}

Es ist jeweils nur eine Aussage pro Auswahl korrekt. Bitte wählen Sie bei allen Aussagen die jeweils richtige. Die Aussagen beziehen sich auf die Artikel S. 16-25 in der Rubrik Spektrum in dieser Ausgabe (DHZ 5/2011).

\section{Welche Aussage trifft jeweils zu?}

\section{Auswahl 1}

a Nahrungsmittelunverträglichkeiten sind das Gleiche wie Nahrungsmittelallergien.

b Nahrungsmittelunverträglichkeiten treten selten Hand in Hand mit -allergien auf.

c Eine Nahrungsmittelallergie bedarf einer signifikanten Allergendosis.

d Bei Nahrungsmittelunverträglichkeiten müssen bereits Spuren des Auslösers gemieden werden.

e Bei Nahrungsmittelallergien ist das betreffende Allergen selbst in geringsten Spuren zu vermeiden.

\section{Auswahl 2}

a Beim Latex-Frucht-Syndrom besteht eine Kreuzallergie z. B. mit Fenchel, Liebstöckel und Ficus.

b Beim Birke-Nuss-Syndrom besteht eine Kreuzallergie z. B. mit Gräsern und Roggen.

c Beim Doldengewächs-Syndrom besteht eine Kreuzallergie z. B. mit Dill, Anis- und Kümmelfrüchten.

d Beim Kuhmilch-Rinderepithelsyndrom besteht eine Kreuzallergie z. B. mit Garnelen.

e Beim Hülsenfruchtsyndrom besteht eine Kreuzallergie z. B. mit Sellerie- und Beifußgewürzen.

\section{Auswahl 3}

a Nahrungsmittelallergien werden durch Immunglobuline vom Typ IgM vermittelt.

b Antikörper vom Typ IgE hemmen die Histaminausschüttung, wodurch die Allergie entsteht.

c Nahrungsmittelallergien können mit Beta-Blockern behandelt werden.

d Nahrungsmittelallergien können mit $\mathrm{H}_{1}$-Blockern behandelt werden.

e Nahrungsmittelallergien können mit Diaminooxidase (DAO) behandelt werden.

\section{Auswahl 4}

a Typischerweise treten die Symptome bei Nahrungsmittelallergien unmittelbar nach der Einnahme auf. b Typischerweise treten die Symptome bei Nahrungsmittelallergien erst nach einiger Zeit in tieferen Abschnitten des Verdauungstrakts auf.

c Das orale Allergiesyndrom tritt typischerweise in Erscheinung mit eosinophiler Ösophagitis, Gastritis oder Colitis.

d Die Diagnose einer Nahrungsmittelallergie erfolgt z. B. mittels ELISA- oder PRICK-Test.

e Ein PRICK-Test kann mit geringem Aufwand risikofrei in der Heilpraktikerpraxis durchgeführt werden.

\section{Auswahl 5}

a Hitzelabile Allergene können sehr gut bei Zubereitung in der Mikrowelle zerstört werden.

b Der häufige Einsatz von Antazida, Basenpulvern und Säureblockern trägt wahrscheinlich zur Zunahme der Nahrungsmittelallergien bei.

c Nahrungsmittelunverträglichkeiten kommen deutlich seltener vor als Nahrungsmittelallergien.

d Laktoseintoleranz findet sich bei ca. $70 \%$ der Bevölkerung Skandinaviens und zu $3 \%$ bei Bewohnern des Mittelmeerraums.

e Eine Fruktosemalabsorption kommt sehr selten vor.

\section{Auswahl 6}

a Heutzutage werden täglich ca. 10-15 g Fruktose mit der Nahrung aufgenommen.

b High Fiber Cereals Syrup (HFCS) ist heutzutage aus der Lebensmittelproduktion nicht mehr wegzudenken.

c Fruktosemalabsorption kann nicht nur zum „Reizdarmsyndrom“, sondern auch zu Depressionen führen.

d Patienten mit Fruktosemalabsorption entwickeln eine Abneigung gegen süße Speisen.

e Eine Reduktion von Fruchtzucker führt zu einer Vitaminunterversorgung.

\section{Auswahl 7}

a Typische Symptome einer Nahrungsmittelunverträglichkeit sind z. B. Bauchschmerzen, Meteorismus, Völlegefühl oder Diarrhöen.

b Eine Nahrungsmittelunverträglichkeit verursacht ausschließlich Symptome im Verdauungstrakt.

c Im Gegensatz zur Nahrungsmittelallergie verursacht eine Nahrungsmittelunverträglichkeit keine Verhaltensauffälligkeiten. d Nur IgE-vermittelte Reaktionen werden als immunologische Nahrungsmittelunverträglichkeit bezeichnet.

e Zu den nicht-immunologischen Reaktionen gehören die pharmakologischen, die pseudoallergischen und die nicht-IgEvermittelten Nahrungsmittelunverträglichkeiten.

\section{Auswahl 8}

a Jeder 5. Deutsche soll eine mehr oder weniger ausgeprägte Kohlehydratintoleranz haben.

b Die Laktoseintoleranz ist in Deutschland die häufigste Nahrungsmittelunverträglichkeit.

c Nahrungsmittelintoleranzen können auch durch einen gestörten Transport verursacht sein (z. B. Fruktose- und Sorbitintoleranz).

d Die Diagnose der Kohlehydratintoleranz wird durch PRICK- oder RAST-Test gestellt.

e Eine Laktoseintoleranz entsteht durch die Freisetzung von $\mathrm{CO}_{2}, \mathrm{H}_{2}$ und kurzkettigen Fettsäuren im Darm.

\section{Auswahl 9}

a Knapp die Hälfte der Weltbevölkerung verliert nach dem Abstillen die Fähigkeit, Laktose aufzuspalten.

b Bis zu $50 \%$ der Menschen mit Laktoseintoleranz leiden auch unter Fruktoseintoleranz.

c Am häufigsten kommt der sekundäre Laktosemangel vor.

d Die Laktoseintoleranz wird heute meist mit einem $\mathrm{H}_{2}$-Atemtest nachgewiesen.

e Leider fällt der $\mathrm{H}_{2}$-Atemtest bei ca. $30 \%$ der Patienten falsch negativ aus.

\section{Auswahl 10}

a Die Fruktoseintoleranz basiert auf einem Enzymmangel.

b Bei Fruktoseintoleranz ist eine völlige Karenz unverzichtbar.

c Eine IgG-Reaktion auf Nahrungsmittel ist immer pathologisch.

d Die IgG4-Untersuchungen sind fachlich unumstritten.

e Die Differenzierung zwischen einer echten Nahrungsmittelallergie und einer unverträglichkeit ist durch das EPX (auch EDN) möglich. 


\section{DHZ-Fortbildungspunkte - Antwortbogen}

\section{DHZ-Antworten}

Lernerfolgskontrolle. Bitte nur eine Antwort pro Auswahl ankreuzen! Sie können in dieser DHZ-Ausgabe 2 Fortbildungspunkte erzielen.

$\begin{array}{lllllll}\text { Frage } & 1 & \text { a } & \text { b } & \text { C } & \text { d } & \text { e } \\ \text { Frage } & 2 & \text { a } & \text { b } & \text { c } & \text { d } & \text { C } \\ \text { Frage } & 3 & \text { a } & \text { b } & \text { C } & \text { d } & \text { e } \\ \text { Frage } & 4 & \text { a } & \text { b } & \text { C } & \text { d } & \text { e } \\ \text { Frage } & 5 & \text { a } & \text { b } & \text { c } & \text { d } & \text { e }\end{array}$

$\begin{array}{llllll}\text { Frage } 6 & \text { a } & \text { b } & \text { C } & \text { d } & \text { e } \\ \text { Frage } 7 & \text { a } & \text { b } & \text { C } & \text { d } & \text { C } \\ \text { Frage } 8 & \text { a } & \text { b } & \text { C } & \text { d } & \text { e } \\ \text { Frage } 9 & \text { a } & \text { b } & \text { C } & \text { d } & \text { e } \\ \text { Frage } 10 & \text { a } & \text { b } & \text { C } & \text { d } & \text { e }\end{array}$

Frage 7 a b c d e

Frage 9 a $b$ c $d$ d

Frage 10 (a) b c d e

\section{Angaben zur Person}

Name, Vorname

Straße

PLZ | Ort

\section{Feld für DHZ-Wertmarke|Abo-Nummer}

Nicht-Abonnenten bitte hier DHZ-Wertmarke aufkleben. Abonnenten bitte Abonummer eintragen.

\section{Erklärung}

Ich versichere, dass ich die Beantwortung der Fragen selbst und ohne Hilfe durchgeführt habe.

$$
\text { Ort | Datum }
$$

Unterschrift

\section{Ihr Ergebnis}

Sie haben

Fragen richtig beantwortet.
Sie haben

DHZ-Punkte erworben.
Sie haben nicht erfolgreich teilgenommen.

Einsendeschluss 29.10.2012 (Datum des Poststempels)

Bitte senden Sie den vollständigen Antwortbogen zusammen mit einem

an Sie selbst adressierten und ausreichend frankierten Rückumschlag an den 
richtseinheiten Heimstudium). Sie stellen sicher, dass die überprüften Therapeuten die klassische Homöopathie in Theorie und Praxis beherrschen. Für Homöopathen nach der Ausbildung ist neben einer Zertifikatsprüfung eine 3-jährige Supervisionszeit obligatorisch. Mit dem Erhalt des Zertifikats verpflichten sich die zertifizierten Homöopathen, ihr Wissen ständig auf dem Laufenden zu halten. Regelmäßige Fortbildungen auf homöopathischem (30 Unterrichtseinheiten à 45 min pro Jahr) und schulmedizinischem Gebiet (8 Unterrichtseinheiten à 45 min pro Jahr) müssen alle 2 Jahre nachgewiesen werden, um die Zertifizierung aufrechtzuerhalten (Infos unter www.homoeopathie-zertifikat.de).

Die SHZ hat sich seit der Ausgabe DHZ 6/2008 entschieden, allen Zertifikatsinhabern die Chance der interaktiven Fortbildung anzubieten, um den Nachweis für die klinische Fortbildung zu erbringen. So können die Zertifikatsinhaber die Fortbildungspunkte der DHZ bei der SHZ als Nachweis für die klinische Fortbildung einreichen (max. 4 Unterrichtseinheiten pro Jahr - das entspricht 4 Fortbildungspunkten der DHZ).

\section{Verbände und unabhängige Stiftung stehen für Qualitåt}

Das Entscheidende an diesen beiden Systemen ist, dass Teilnehmer nicht einfach Teilnahmebescheinigungen von Fortbildungen sammeln können, sondern dass eine Zertifizierungsstelle, hier ein Berufsverband bzw. eine Stiftung, für die Qualität der Fortbildung steht und diese überprüft und dokumentiert.

Das DHZ-Team unterstützt den Ansatz der regelmäßigen qualifizierten und dokumentierten Fortbildung. Die interaktive Fortbildung halten wir für ganz besonders geeignet, eine qualifizierte Fortbildung zu belegen, weil hier eine eindeutige Lernerfolgskontrolle besteht. Nur wer die Fragen richtig beantwortet, erhält auch seine Fortbildungspunkte.

\section{So geht's}

Sie finden in jeder DHZ-Ausgabe Fortbildungseinheiten. Diese können Sie - auch unabhängig von der Zertifizierung - regelmäßig nutzen, um Ihren Lernerfolg zu überprüfen und zu dokumentieren. Andererseits können Sie sich diese DHZ-Fortbildungs- punkte auch für die Fortbildungszertifikate der genannten Verbände anerkennen lassen.

\section{Anerkennung der DHZ-Beitråge}

Die Vergabe der Fortbildungspunkte kann direkt durch die DHZ erfolgen.

Pro Lerneinheit erhalten Sie 2 Fortbildungspunkte. Hierfür:

müssen 7 von 10 Fragen richtig beantwortet sein.

nuss der Antwortbogen vollständig ausgefüllt sein.

-1 muss im entsprechenden Feld des Antwortbogens eine DHZ-Wertmarke aufgeklebt oder Ihre DHZ-AboNummer eingetragen sein.

Die Abo-Nummer finden Sie auf dem Adressaufkleber Ihres Heftes.

\section{Beantworten und abschicken!}

$\mathrm{Zu}$ einzelnen Beiträgen der $\mathrm{DHZ}$ gibt es in jeder Ausgabe Fragebögen (S. II), die Sie ab 2010 auch im Internet unter www.thiemeconnect.de/ejournals/toc/dhz (auch als Nicht-Abonnent der DHZ) herunterladen und ausdrucken können. Schicken Sie uns einfach den ausgefüllten Antwortbogen (S. III). Wir prüfen Ihre Antworten und benachrichtigen Sie über Ihren Lernerfolg. Die Bescheinigung können Sie dann bei der zentralen Dokumentationsstelle des Fortbildungszertifikats für Heilpraktiker beim BDH und für das Homöopathiezertifikat bei der SHZ einreichen.

Die Teilnahme ist rückwirkend bis max. 1 Jahr nach Erscheinen der jeweiligen DHZ-Ausgabe möglich. Beachten Sie den Einsendeschluss auf dem Antwortbogen.

\section{Datenschutz}

Ihre Daten werden ausschließlich für die Bearbeitung dieser Fortbildungseinheit verwendet. Es erfolgt beim Verlag keine Speicherung der Ergebnisse über die für die Bearbeitung der Fortbildungseinheit notwendige Zeit hinaus. Die Daten werden nach Versand der Punktbescheinigungen anonymisiert. Namen- und Adressangaben auf den Antwortbögen dienen ausschließlich dem Versand der Punktbescheinigungen. Die Angaben zur Person dienen nur statistischen Zwecken und werden von den Adressangaben getrennt und anonymisiert verarbeitet.

\section{DHZ-Wertmarke für}

\section{Nichtabonnenten}

DHZ-Wertmarken können beim Verlag erworben werden: 6er-Pack DHZ-Wertmarken; Preis 25,00 € inkl. MwSt.; Artikel-Nr. 902999

\section{Bitte richten Sie Ihre Bestellung an: MVS Medizinverlage Stuttgart KundenServiceCenter Buch Postfach 301120; 70451 Stuttgart Tel.: 0711/8931-900}

Dieser Artikel ist online zu finden unter: http://dx.doi.org//10.1055/s-0031-1293561

\section{Teilnahme}

Jeder Heilpraktiker soll die Möglichkeit zur Teilnahme an der Fortbildung erhalten. Deshalb ist die Teilnahme an der DHZ-Fortbildung nicht an ein Abonnement geknüpft. Dennoch sollten Sie im Besitz einer $\mathrm{DHZ}$ sein, denn alle im Fragebogen enthaltenen Fragen beziehen sich auf die Spektrumbeiträge der jeweiligen Ausgabe. An diversen Heilpraktikerschulen finden Sie DHZExemplare, die dort ausliegen. Darüber hinaus können Sie als Nicht-Abonnent der DHZ Einzelexemplare erwerben bei: KundenServiceCenter Zeitschriften, Rüdigerstr. 14, 70469 Stuttgart, Tel.: 0711/8931-308. Für Abonnenten ist die Teilnahme kostenfrei. Nicht-Abonnenten können DHZ-Wertmarken erwerben. 